\title{
El embarazo adolescente en Quintana RoO: DEBATES y APORTES DE INVESTIGACIÓN PARA POLÍTICAS PÚBLICAS DE PREVENCIÓN Y ATENCIÓN
}

\section{Adolescent Pregnancy in Quintana Roo: Debates and Research Contributions for Public Policies on Prevention and Care}

\author{
Alejandra Pamela España-Paredes* \\ Leticia Janet Paredes-Guerrero** \\ Rocío Ivonne Quintal-López***
}

Resumen: El objetivo de este artículo es contrastar cómo se entiende el embarazo adolescente desde el Estado y sus políticas públicas, y desde adolescentes y sus familias que se encuentran en condiciones de vulnerabilidad. Se basa en trabajo de campo con mujeres embarazadas de entre 12 y 19 años de tres municipios del estado de Quintana Roo. Si bien el embarazo adolescente presenta grandes riesgos para la salud de la madre y del neonato, así como impactos sociales y psicológicos, los contextos sociales y culturales encaminan a verlo como parte de lo esperado en los proyectos de vida de las adolescentes. Por ello, las políticas públicas deben responder a contextos socioeconómicos inmediatos y a la condición de género para poder generar estrategias reales encaminadas a la disminución de esta situación.

Palabras clave: jóvenes, embarazo, condición de género, salud pública, políticas públicas.

Abstract: The objective of this article is to contrast how the state and public policies understand adolescent pregnancy when compared to views held by adolescents and their families in vulnerable conditions. The article is based on fieldwork with pregnant women aged 12 to 19 from three municipalities in the state of Quintana Roo. Adolescent pregnancy has great risks to the health of mothers and newborns, as well as social and psychological impacts. Thus, in order to reduce pregnancy within this group, public policies should be prepared in a way that responds to adolescents' immediate socio-economic context and gender status.

Keywords: youth, pregnancy, gender condition, public health, public policies.

\footnotetext{
* Alejandra Pamela España Paredes. Doctora en Educación por la Universidad del Sur, campus Mérida, México. Profesora investigadora en la Unidad de Ciencias Sociales del Centro de Investigaciones Regionales Dr Hideyo Noguchidela Universidad Autónoma de Yucatán, México. Temas de especialización: estudios de género, antropología, pobreza urbana en mujeres, violencia y derechos humanos. Correo electrónico: alejandra.espana@correo.uady.mx, ORCID: https:/orcid. org/0000-0002-3814-8065.

** Leticia Janet Paredes Guerrero. Doctora en Conocimiento y Cultura en América Latina por el Instituto Pensamiento y Cultura en América Latina (IPECAL), México. Profesora investigadora en la Unidad de Ciencias Sociales del Centro de Investigaciones Regionales Dr Hideyo Noguchi de la Universidad Autónoma de Yucatán, México. Temas de especialización: estudios de género, participación política
}

de las mujeres, transversalidad de la perspectiva de género, violencia y discriminación en universidades. Correo electrónico: guerrero@correo. uady.mx. ORCID: https:/orcid.org/0000-0002-6457-7955.

*** Rocío Ivonne Quintal López. Doctora en Ciencias Sociales por la Universidad Autónoma Metropolitana-Xochimilco, México. Profesora investigadora en la Unidad de Ciencias Sociales del Centro de Investigaciones Regionales Dr Hideyo Noguchi de la Universidad Autónoma de Yucatán, México. Temas de especialización: género, sexualidad y Derechos Humanos. Correo electrónico: rocio.lopez@ correo.uady.mx. ORCID: https://orcid.org/0000-0003-3879-3360.

Enviado a dictamen: 2 de febrero de 2018

Aprobación: 6 de noviembre de 2018

Revisiones: 1 
AlejandRa PAmela EsPaña-PAREdes, LETICIA JANET PAREDES-GUERRERO, ROCÍO IVONNE QUINTAL-LÓPEZ

\section{Introducción}

A nivel mundial, agencias y organismos internacionales consideran el embarazo adolescente como un problema de salud pública relevante porque las adolescentes constituyen el grupo poblacional que presenta mayor riesgo de mortalidad materna y porque las complicaciones durante el embarazo y el parto son la segunda causa de muerte en adolescentes en el mundo - 16 millones de niñas de entre 15 y 19 años son madres cada año-. Los bebés nacidos de madres adolescentes representan el $11 \%$ de los nacimientos, y el 95\% de ellos viven en países en vías de desarrollo (OMS, 2014; Naciones Unidas, 2015).

En la V Conferencia de Población y Desarrollo, que se llevó a cabo en El Cairo en 1994, se reconoció y definió el derecho humano a la salud reproductiva, entendida ésta como el derecho básico de todas las personas y parejas a decidir libre y responsablemente el número de hijos que desean tener y el espaciamiento de los nacimientos, así como a adoptar decisiones relativas a la reproducción sin sufrir discriminación, coacciones o violencia (Lassonde, 1997; Naciones Unidas, 1995). Más de 180 países, incluido México, se comprometieron a tomar medidas para salvaguardar los derechos reproductivos utilizando mecanismos como la promoción de acciones dirigidas a fomentar las condiciones de igualdad entre mujeres y hombres y a ofrecer métodos anticonceptivos adecuados y gratuitos, programas de orientación y prevención del embarazo adolescente, y servicios de salud accesibles para que las parejas cuenten con las máximas posibilidades de tener hijos sanos y embarazos y partos sin riesgos.

Entre 1990 y 2015, la tasa de fecundidad adolescente en México disminuyó, al pasar de 83 a 64 nacimientos por mil mujeres. Si bien son menos las mujeres adolescentes que se embarazan, de 1994 a 2015 el porcentaje de adolescentes embarazadas con respecto al total de mujeres en el país pasó del $16.9 \%$ al $18.2 \%$, lo que significó un aumento del 1.3\% (Banco Mundial, 2015; INEGI, 2015). A pesar de los compromisos adquiridos hace más de quince años, México no cumplió con uno de los objetivos de desarrollo del milenio ya que ocupa
EL EMBARAZO ADOLESCENTE EN QUINTANA ROO: DEBATES Y APORTES DE INVESTIGACIÓN PARA POLÍTICAS PÚBLICAS DE PREVENCIÓN Y ATENCIÓN

el primer lugar en embarazo adolescente entre los países de la Organización para la Cooperación y el Desarrollo Económicos (OCDE), puesto que uno de cada cinco embarazos se presenta en jóvenes menores de edad. El mismo organismo indica que la cifra creció del 30\% al $37 \%$ de 2005 a 2011 (OCDE, 2017).

En este escenario, se han llevado a cabo importantes estudios en diversas disciplinas como la biomédica, la psicología social, la antropológica, la sexología, la sociodemografía y los estudios poblacionales, principalmente en torno a las relaciones sexuales entre adolescentes, en temas como la edad de inicio de las relaciones, la frecuencia, el número de parejas, el conocimiento, acceso y uso real de métodos anticonceptivos o la adquisición de infecciones de transmisión sexual (Núñez y Ayala, 2012; Vélez, 2012; Okumura et al. 2014). La comunidad académica también ha caracterizado el comportamiento sexual y reproductivo de las adolescentes como un problema de salud pública, que a su vez genera un perfil epidemiológico muy específico debido a las consecuencias adversas que conllevaría para éstas, sus familias y las comunidades (Stern, 2003; Zamudio et al., 2017).

Como muestran las cifras de la OMS, la probabilidad de que una mujer de 15 años fallezca por una causas maternas es de l en 4900 en los países desarrollados y de 1 en 180 en los países en desarrollo, una brecha muy amplia asociada a las condiciones y a los accesos de los servicios de salud. Por lo anterior, el embarazo y el parto entre adolescentes es un factor de riesgo grave que lleva a complicaciones obstétricas y perinatales que pueden terminar con la vida de la madre y el bebé. Además, desde la visión del Estado el embarazo de niñas menores de edad está relacionado con la perpetuación del ciclo de la pobreza, la deserción escolar, la violencia intrafamiliar y el aumento de su vulnerabilidad en diversos ámbitos. Estas consecuencias de tipo social las viven principalmente las madres adolescentes, que con frecuencia abandonan sus estudios para dedicarse al hogar y el cuidado de los hijos. Esto les lleva a privarse de una formación educativa y a limitar sus posibilidades laborales, lo que se vuelve un impedimento para 
ALEJANDRA PAMELA ESPAÑA-PAREDES,

mejorar las condiciones económicas y sociales de sus familias y las torna dependientes del ingreso de sus parejas o familias (Reyes y González, 2014). Desde una aproximación psicosocial, muchos estudiosos han evidenciado el hecho de que el embarazo durante la adolescencia trunca posibilidades de desarrollo personal y conduce a la pérdida de proyectos de vida debido a las consecuencias antes mencionadas (Vélez, 2012).

Los Objetivos de Desarrollo Sustentable aseguran que es posible la prevención del embarazo a temprana edad mediante una educación integral en sexualidad, de calidad óptima, que incluya contenidos de género y que asegure establecer vínculos con los servicios de salud para garantizar el acceso a anticonceptivos. A su vez, se espera que los países firmantes implementen de manera apropiada políticas públicas que conduzcan a la reincorporación de las adolescentes a la escuela, garantizando que ejerzan su derecho a la educación (UNESCO, 2017).

No obstante, miles de niñas y adolescentes se embarazan y dan a luz cada año en este país, lo que incluso ha llegado a normalizarse. En estudios empíricos como los de Claudio Stern (1997; 2003) se ha mostrado que la construcción del "problema" del embarazo adolescente por parte del Estado y de agencias internacionales puede diferir ampliamente de las concepciones de los propios adolescentes y sus familias, que a su vez cambian dependiendo del grupo o sector social en el que se encuentren y de cada contexto específico. Es decir, en su imaginario, muchos jóvenes - y en esto coinciden los de distintos estratos sociales- ven el embarazo, la vida en pareja y la familia como algo esperado por el contexto social, muchas veces independientemente de la edad en la que llegue este evento. En las familias mexicanas aún se sostienen una serie de valores que privilegian la llegada al mundo de un hijo a temprana edad sobre el uso de anticonceptivos o la opción del aborto. En otros casos, las vidas de las niñas y adolescentes se ven cruzadas por la falta de información en torno a la sexualidad y a la violencia sexual y de género, lo que les deja poco margen de acción sobre su propio cuerpo, salud y reproducción. En estos
EL EMBARAZO ADOLESCENTE EN QUINTANA ROO: DEBATES Y APORTES DE INVESTIGACIÓN PARA POLÍTICAS PÚBLICAS DE PREVENCIÓN Y ATENCIÓN

y otros casos, las ideas, significados y prácticas en torno a la salud sexual y reproductiva complejizan el tema del embarazo adolescente, más allá de considerarse un asunto de prevención en salud pública. Además, desde un enfoque de género no puede ignorarse que la maternidad es una expectativa que la sociedad deposita en las mujeres de manera profunda, por lo que el embarazo a temprana edad puede ser también una consecuencia de la condición de género que las adolescentes se ven de alguna manera obligadas a asumir.

Por ello, vale la pena preguntarse, desde un contexto situado, si el embarazo adolescente es una problemática, en qué sentidos y para quiénes. Así, el objetivo del presente artículo es contrastar la construcción que el Estado hace del embarazo adolescente a través de sus políticas públicas, con la que realizan las propias adolescentes y sus familias en condiciones de vulnerabilidad del estado de Quintana Roo, desde su realidad cotidiana y de acuerdo con su propia concepción. Para ello, exploramos los cambios en diversos aspectos de la vida de jóvenes que fueron madres a temprana edad, antes, durante y después de su embarazo. Sostenemos que esta aproximación ofrece pistas empíricas importantes para generar políticas públicas adecuadas enfocadas en la prevención del embarazo adolescente y en la promoción de la salud reproductiva entre los jóvenes.

\section{El embarazo adolescente en Quintana Roo, caracterización de un contexto situado}

El 59.6\% de la población femenina de Quintana Roo se encuentra en edad fértil, lo que significa que 389000 mujeres están en posibilidad de ser madres (COESPO, 2013). En el caso específico de la fecundidad en adolescentes de 15 a 19 años, el estado ocupa un lugar importante por encima del promedio nacional puesto que, según el censo de 2010, los nacimientos en mujeres de este grupo de edad eran del $60.66 \%$ por cada mil habitantes, frente al promedio nacional del $56.86 \%$ (INEGI, 2015). De acuerdo con la Encuesta Nacional de Salud y Nutrición, el 23.1\% de las mujeres inicia 
AlejandRa PAMELA EsPaña-PAREDES, LETICIA JANET PAREDES-GUERRERO, ROCÍO IVONNE QUINTAL-LÓPEZ

su vida sexual entre los 12 y 19 años, es decir, durante la adolescencia, ${ }^{1}$ y es relevante notar que de aquellas que ya han iniciado su vida sexual, el $47.6 \%$ ha estado embarazada alguna vez (ENSANUT, 2012).

En una encuesta realizada en 2009 en Quintana Roo, el 28.9\% de las mujeres consultadas dijo no haber planeado su embarazo, mientras que el 16.3\% mencionó que era no deseado. De las mismas encuestadas, el 54.9\% de las embarazadas afirmó haber planeado su situación (COESPO, 2013). En 2011, 9105 mujeres casadas dieron a luz y 13399 lo hicieron viviendo en unión libre. Por su parte, el $12.21 \%$ de las mujeres que se embarazaron se encontraban solteras (COESPO, 2011).

Quintana Roo es un estado cuyo desarrollo económico, poblacional y de servicios educativos y de salud ha crecido exponencialmente desde la década de los noventa del siglo XX. Debido a su fuerte orientación en el sector servicios, específicamente en el turismo, de acuerdo con el Consejo Nacional de Evaluación de la Política de Desarrollo Social (CONEVAL, 2012) la entidad ocupa apenas el lugar 26 de las 32 del país en cuanto a porcentaje de pobreza entre su población, y el 21 con respecto a pobreza extrema. Por ello, Quintara Roo recibe una cantidad importante de migrantes nacionales provenientes de la península de Yucatán y del centro del país para ocuparse en el turismo, sector que genera miles de trabajos informales, temporales y de baja calificación.

En general el analfabetismo en el estado se encuentra por debajo de la media nacional, con sólo el 4.86\% de analfabetos menores de 15 años (COESPO, 2010). Respecto a los grados de escolaridad, Quintana Roo ocupa un lugar privilegiado en el país, ya que se encuentra en el noveno lugar en cuanto a población con más número de grados cursados -9.1 años, mientras el promedio nacional es de 8.6 - (INEGI, 2010). Sin embargo, en el caso de la educación básica, la entidad se encuentra por debajo de la media, mientras que sobresale en educación media superior. Por otra parte, en educación superior el estado se encuentra prácticamente a la par del promedio del país: el 16\% de la población tiene acceso a este grado, mientras que la media del país es del 16.5\%.
EL EMBARAZO ADOLESCENTE EN QUINTANA ROO: DEBATES Y APORTES DE INVESTIGACIÓN PARA POLÍTICAS PÚBLICAS DE PREVENCIÓN Y ATENCIÓN

El presente artículo se basa en trabajo de campo realizado en el año 2013 en las tres cabeceras municipales del estado donde se detectó el mayor número de embarazos en adolescentes: Cancún, en el municipio de Benito Juárez; Playa del Carmen, en Solidaridad, y Chetumal, en Otón P. Blanco. Se realizó un estudio cuantitativo de tipo exploratorio en el que se determinó una muestra a partir de un diseño no probabilístico de cuota del 10\% de las mujeres ubicadas entre los 12 y los 19 años que habían tenido por lo menos un hijo nacido vivo, cifra que según el Censo de Población y Vivienda (INEGI, 2010) ascendía a 5997 mujeres. Para la muestra se determinó a un total de 583 mujeres distribuidas en las tres principales ciudades: 191 en Chetumal, 209 en Cancún y 183 en Playa del Carmen. Se determinó entrevistar a mujeres de entre 12 y 19 años que hubieran estado embarazadas en los últimos tres años (20102013). Debido a que el censo utilizado para generar la muestra fue de 2010, identificamos que las mujeres susceptibles a entrevistar durante el censo contaban entre 12 y 18 años de edad, pero cuando se aplicó la encuesta en 2013 dichas mujeres ya tenían entre 15 y 21 años. Esta situación originó que incluyéramos dentro de la muestra a mujeres que, si bien en 2013 ya tenían 21 años, en 2010 contaban con 18 o 19 años y se encontraban embarazadas de su primer o segundo hijo, por ello no rebasamos el rango de edad establecido para el estudio y explica por qué en los datos recolectados se encuentran mujeres con 21 años de edad.

Se utilizó un corte mixto a partir de la construcción de un instrumento único que consistió en una encuesta semiestructurada aplicada en escuelas, centros de salud, instituciones de gobierno y domicilios particulares. En dicho instrumento se exploraron las siguientes variables: estructura familiar y relaciones interpersonales, conocimiento sobre sexualidad, prácticas sexuales y uso de métodos anticonceptivos, vida cotidiana, autoestima y plan de vida, percepciones sobre ser mujer y embarazo, así como políticas públicas y servicios; a su vez, y de manera transversal, se cruzaron dichas variables por dos dimensiones dadas a partir de la condición de embarazo en adolescentes: antes y después del embarazo, que se traducen en las causas y los efectos 
ALEJANDRA PAMELA ESPAÑA-PAREDES,
EL EMBARAZO ADOLESCENTE EN QUINTANA ROO: DEBATES Y APORTES DE INVESTIGACIÓN PARA POLÍTICAS PÚBLICAS DE PREVENCIÓN Y ATENCIÓN del mismo y de las cuales se describen algunas en el presente artículo (ver Tabla 1).

A continuación discutiremos brevemente el embarazo adolescente a partir de la condición de género y como factor de vulnerabilidad entre las mujeres jóvenes, como marco de análisis para observar las diferencias entre la construcción del embarazo adolescente como problema por parte del Estado y sus políticas públicas, y la concepción del mismo por parte de las madres adolescentes y sus familias. El siguiente apartado constituye una aproximación teórica y normativa sobre los principales supuestos que han caracterizado al embarazo adolescente como problema de salud pública con consecuencias económicas, sociales y de salud para las adolescentes, sus hijos y sus familias en general. Posteriormente se presentan los hallazgos de la investigación y se analizan las concepciones del embarazo entre mujeres que vivieron este proceso durante su adolescencia, el funcionamiento y el rol que jugaron sus redes de apoyo y contención, y las implicaciones que tuvo el embarazo en su educación y en su proyecto de vida. Para finalizar, en las conclusiones se contrastarán las construcciones del embarazo adolescente entre los diferentes actores y se propondrán algunas pistas para replantear las políticas públicas de prevención del embarazo.

\section{El embarazo adolescente como condición de género}

Consideramos el embarazo adolescente como aquel que sucede antes de los 19 años de edad, tal y como se establece en el Modelo de Atención Integral en Salud Sexual y Reproductiva para Adolescentes y en el Programa de Acción Específico Salud Sexual y Reproductiva de Adolescentes, aunque vale la pena destacar que la antropología ha documentado en extenso que la adolescencia se significa y experimenta de manera distinta en las diversas culturas y sectores sociales (Reyes y González, 2014). Stern (2003) menciona que incluso las consecuencias emocionales y sociales del embarazo a temprana edad son distintas de acuerdo con el grupo social de las mujeres. Las jóvenes de sectores más empobrecidos suelen sufrir más efectos negativos en su salud y economía, mientras que las de estratos medios y altos experimentan un mayor impacto en sus proyectos de escolaridad prolongada, además de que cuentan con la opción - legal o no-de interrumpir su embarazo. En este hecho se basa la tesis del autor que consiste en no considerar el embarazo adolescente como un problema homogéneo y generalizable a priori, sino como un fenómeno social cuyas causas e impactos varían de acuerdo con cada individuo, comunidad y sociedad.

Sin embargo, a pesar de que las situaciones que rodean a cada mujer varían y las afectan de diversas maneras, existe una "condición de género" que es común a todas ellas sin importar su estrato social. Por condición de género nos referimos a los mecanismos sociales, económicos y culturales en que desarrollan su existencia las mujeres en comparación con los hombres, lo que las pone en situación de desventaja y subordinación. Esta condición se expresa en una serie de mecanismos de apropiación y control de su cuerpo, reproducción y linaje, que en la vida cotidiana se materializan en la educación y en la presión social para cumplir ciertos roles y expectativas determinados para el género femenino. Diversas autoras feministas han argumentado que la heterosexualidad, el matrimonio, la maternidad y la familia son algunos de los elementos más importantes de la dominación patriarcal que se inculcan a las mujeres. Ser madre es sin duda uno de los mandatos de género más ampliamente fomentado en la mayoría de las sociedades, siendo incluso justificado desde la ciencia, naturalizado y presentado como la "esencia femenina", como si las mujeres tuvieran un instinto biológico irracional por reproducirse y una proclividad natural al cuidado y protección de los otros (Rosso, 2016).

Así, observamos que para las mujeres encuestadas independientemente de la situación y del contexto social en que viva cada una, ser pareja de un hombre, ser madre y formar una familia son algunos de los preceptos fundamentales que dan estructura a su proyecto de vida y contenido a sus sueños, expectativas y deseos. Cuando las niñas y adolescentes no tienen la oportunidad 
AlejandRa PAMELA EsPaña-PAREDES, LETICIA JANET PAREDES-GUERRERO, ROCÍO IVONNE QUINTAL-LÓPEZ
EL EMBARAZO ADOLESCENTE EN QUINTANA ROO: DEBATES Y APORTES DE INVESTIGACIÓN PARA POLÍTICAS PÚBLICAS DE PREVENCIÓN Y ATENCIÓN de elegir y construir un proyecto de vida autónomo, tenderán a reproducir aquel en el que han sido socializadas como natural e ineludible. Sin importar si sucede antes o después, la maternidad es considerada como un destino, una obligación y un deseo. Aunque el embarazo entre las jóvenes entrañe complicaciones en su salud y en la economía de sus familias, la sociedad considera que las mujeres están cumpliendo con su condición de género. Así, la maternidad funciona como un mecanismo de escape a la incertidumbre de la adolescencia ante la falta de un proyecto de vida y coloca a las mujeres en su rol predeterminado social y "naturalmente". Además, aunque las mujeres tengan acceso a información y a métodos anticonceptivos para evitar un embarazo no deseado, la desigualdad de poder entre ellas y sus parejas varones socaba su autonomía para tomar decisiones sobre su salud reproductiva, lo que las expone a coacción sexual y a diversas formas de violencia, tal como se ha presentado en diversos estudios en los que se ha demostrado que las barreras para el acceso a métodos anticonceptivos están relacionadas con tabúes y presiones sociales y de género que limitan a las jóvenes la regulación natal (Menkes y Suárez, 2003).

De esta manera, la condición de género se torna un factor de vulnerabilidad que se suma a otras vulnerabilidades de origen social, económico, étnico, racial o etario. Es por ello que las mujeres se consideran los sujetos sociales más vulnerables de cualquier grupo social, más aún si son jóvenes, pobres, indígenas o discapacitadas.

\section{La construcción del embarazo adolescente desde la mirada del Estado y las políticas públicas}

Desdelalógica macrodelEstado, en términosestadísticos y de efectividad de las políticas públicas, un aumento en la fertilidad en las mujeres adolescentes le "resta puntos" al país en el escrutinio internacional, donde se dicta que el número de adolescentes embarazadas debe disminuir de manera pronta con el fin de que el desarrollo personal, profesional y de calidad de vida de ellas aumente, para reflejar así un incremento en el empoderamiento femenino y en el desarrollo económico y social del país.
De lo contrario, seguirán siendo altos los índices de muerte de mujeres embarazadas a temprana edad, la deserción escolar, el acceso a trabajos mal remunerados y la pobreza sistemática. Es decir, desde la mirada del Estado, el embarazo adolescente es un factor crucial para la deserción escolar y para la reproducción del ciclo de pobreza entre las poblaciones más vulnerables.

Desde la firma de los acuerdos del Cairo en 1994, en la Conferencia Internacional sobre la Población y el Desarrollo, la deserción escolar ha sido una de las problemáticas más evidentes relacionadas con el embarazo y la maternidad en la adolescencia, y una de las consecuencias más difíciles de combatir de forma efectiva. Se ha demostrado que el sistema educativo mexicano es altamente discriminatorio y excluyente, tanto para hombres como para mujeres, ya que los grupos más pobres tienen menos acceso a la educación que los que tienen mayores ingresos (Climent, 2003). En México, la población femenina presenta los niveles más altos de rezago y deserción escolar, lo cual se asocia a situaciones de precariedad económica y exclusión social, aunado al hecho de que se sigue promoviendo un patrón cultural según el cual se valora menos la escolaridad de las mujeres, y más su papel en el ámbito doméstico y reproductivo (De Jesús y Menkes, 2011).

Así, muchas jóvenes abandonan la escuela por motivos económicos o familiares incluso antes de su primer embarazo, lo que a su vez las condiciona para vivir embarazos a temprana edad. Otras dejan la escuela al resultar embarazadas o durante los primeros años de sus hijos. Estos datos muestran que el convertirse en madre durante la adolescencia supone, per se, una mayor vulnerabilidad para ellas y sus hijos, lo que a su vez provoca que en la mayoría de los casos interrumpan sus proyectos de vida, entre ellos la trayectoria académica, lo que las predispone a un futuro con un sinfín de limitaciones en los ámbitos laboral, personal y familiar (Rangel et al., 2004; Stern, 2012).

Los puntos anteriores reafirman que en el sistema económico neoliberal en que se encuentra sumergido México, el embarazo adolescente y la deserción escolar son factores para la reproducción del ciclo intergeneracional de la pobreza. Los jóvenes expulsados 
AlejandRa PAMELA EsPaña-PAREDES,

del sistema educativo ven limitada su posibilidad de obtener un empleo bien remunerado, lo cual a su vez restringe el acceso a recursos que permitan un desarrollo adecuado de sus hijos, de modo que se perpetúa un círculo vicioso cuya principal consecuencia es la reproducción de la pobreza entre las generaciones y de las desigualdades de género al interior de las familias. Además, hay que mencionar que la escuela es el espacio donde los jóvenes deben recibir educación sexual formal, laica y científica, como parte de sus derechos humanos y constitucionales, puesto que el Estado no puede garantizar que en la familia se cumpla esta función educativa y es común que los jóvenes no se acerquen a los centros de salud para obtener orientación e información respecto a su sexualidad.

Lo anterior se encuentra plenamente descrito en la Encuesta nacional de la dinámica demográfica 2014 en México, la cual muestra que aproximadamente el 60\% de las mujeres de entre 15 y 19 años que se embarazaron pertenecían a estratos socioeconómicos bajos, que más de la mitad de ellas ya vivía en unión libre o estaba casada, y que más del 60\% ya había dejado la escuela al momento del embarazo (INEGI, 2014). Varios estudios muestran que un fenómeno similar ocurre en general en América Latina, donde el embarazo temprano se asocia a una situación menos ventajosa en términos de bienestar material y social (Climent, 2003; Okumura et al., 2014).

Lo anterior indica que es necesario cuestionar si la afirmación "el embarazo en adolescentes conlleva pobreza" es adecuada o debería reconsiderarse, tomando en cuenta factores como si la mayoría de las adolescentes eran o no pobres antes de quedar embarazadas y si es la pobreza o la falta de oportunidades educativas lo que las orilla al embarazo temprano y las condiciona a enfrentarse a la maternidad con menos recursos económicos y sociales, y con la carga de una doble o triple jornada por el trabajo que realizan al interior del hogar (Golovanevsky, 2006).

El gobierno de la República Mexicana no es ajeno al problema presentado, ya que en 2015 el presidente Enrique Peña Nieto presentó la Estrategia Nacional para la Prevención del Embarazo en Adolescentes (Secretaría de Gobernación, 2015) como política
EL EMBARAZO ADOLESCENTE EN QUINTANA ROO: DEBATES Y APORTES DE INVESTIGACIÓN PARA POLÍTICAS PÚBLICAS DE PREVENCIÓN Y ATENCIÓN

nacional enfocada a reducir a la mitad el número de embarazos en adolescentes de 15 a 19 años de edad y erradicar el embarazo en niñas menores de 15 años, con enfoque de género y respeto a los derechos humanos, particularmente los derechos sexuales y reproductivos, para el año 2030. La estrategia vincula a varias secretarías e institutos nacionales - como la Secretaría de Gobernación, la Secretaría de Desarrollo Social, la Secretaría de Salud, la Secretaría de Educación, el Instituto Nacional de las Mujeres, el Instituto Mexicano de la Juventud, la Comisión Nacional para el Desarrollo del Pueblos Indígenas y el Sistema Nacional para el Desarrollo Integral de la Familia, entre otrasy pretende incidir en todos los niveles de la actuación pública - nacional, estatal y municipal-, con corresponsabilidad ciudadana y participación juvenil. De acuerdo con la Encuesta Nacional para la Prevención del Embarazo en Adolescentes, los ejes principales de la estrategia buscan lograr la permanencia de las mujeres adolescentes en el sistema educativo hasta el último grado obligatorio como mínimo; implementar campañas constantes para generar un entorno en el que las y los adolescentes se sientan cómodos para solicitar métodos de prevención del embarazo y que a su vez cuenten con toda la información para poder utilizarlos $y$, entre otras cosas, invertir en infraestructura para que se pueda acceder a dichos servicios (Secretaría de Gobernación, 2015).

A su vez, el Modelo de Atención Integral en Salud Sexual y Reproductiva para Adolescentes establece que el reto del gobierno federal es impulsar un cambio cultural, con el propósito de que las y los adolescente sean verdaderos protagonistas en el ejercicio de sus derechos sexuales y reproductivos, esto bajo dos premisas principales: la primera, a través de su participación activa en el diseño y operación de estrategias diferenciadas para atender sus necesidades específicas en materia de salud sexual y reproductiva; y la segunda, eliminando los factores que impiden el ejercicio pleno de la sexualidad y de la salud reproductiva de la población adolescente particularmente en áreas rurales e indígenas (Secretaría de Salud, 2014). Esto último no se logró, al menos en la 
AlejandRa PAMELA EsPaña-PAREDES, LETICIA JANET PAREDES-GUERRERO, ROCÍO IVONNE QUINTAL-LÓPEZ

población encuestada, ya que cuando se les interrogó sobre si habían hablado con alguien sobre su sexualidad sólo el 33.4\% respondió afirmativamente, mientras que el 64.9\% indicó que no lo había hecho, lo cual indica que la mayoría de las mujeres no acuden a preguntar sobre sus dudas o temores, lo que les conduce a contar con información sesgada, lo que va en contraposición de una política pública federal de apertura. Asimismo, cuando se les cuestionó sobre con quién hablaron de sexualidad antes de embarazarse, las respuestas de las participantes se agruparon en tres categorías, que por orden de frecuencia son: madre, amigas o compañeras del colegio; hermanas o primas; y médicos o profesores. Estos últimos son considerados fundamentales de acuerdo con el Modelo de Atención Integral en Salud Sexual y Reproductiva para Adolescentes.

\section{Resultados: la construcción del embarazo adolescente desde el seno familiar}

\section{Concepciones del embarazo entre las mujeres adolescentes de Quintana Roo}

Las mujeres quintanarroenses encuestadas, que vivieron un embarazo durante su adolescencia, expresaron varias ideas en torno al tema. Una opinión reiterada fue que vivir un embarazo siendo adolescente (ver Tabla 2) no es malo para la salud; que ser madre a edad temprana no dificulta continuar estudiando, y que no se trata de un problema social. Sin embargo, cuando se les entrevistó respecto a su proyecto de vida, mencionaron que no contemplaban embarazarse a edad temprana y sí visualizaban las consecuencias de tener relaciones sexuales. También mencionaron expresiones como: "nadie te obliga a tener relaciones sexuales", y la mayoría expresó que ellas mismas las aceptaban durante su adolescencia y que sabían que el uso de anticonceptivos era la mejor forma de prevenir el embarazo; a pesar de ello, no los utilizaron.

Es claro que contaban con información para prevenir un embarazo no deseado, pero éste no lo concebían como un riesgo para la salud ni como un obstáculo en su vida - por ejemplo, para seguir estudiando—. También
EL EMBARAZO ADOLESCENTE EN QUINTANA ROO: DEBATES Y APORTES DE INVESTIGACIÓN PARA POLÍTICAS PÚBLICAS DE PREVENCIÓN Y ATENCIÓN

se refirieron a él como una consecuencia lógica y natural de tener relaciones sexuales, como si no fuera posible evitarlo. Además, a pesar de que en la actualidad es fácil obtener información, existen muchos mitos y creencias erróneas en torno al embarazo adolescente que se exacerban en ambientes como en el contexto estudiado, principalmente por falta de educación formal.

Esto permite evidenciar el hecho de que las acciones implementadas por las adolescentes y sus parejas, si bien no fomentan el embarazo, tampoco ayudan a prevenirlo, puesto que no toman medidas concretas para evitarlo. En general, se observó que las entrevistadas conocían las repercusiones de tener relaciones sexuales no protegidas, pero no abundaron en las consecuencias del embarazo ni en las implicaciones que esto tuvo para el resto de sus vidas. Este desconocimiento obedece a distintas situaciones, entre las que cobra más importancia la falta de comunicación entre las adolescentes y sus padres con relación a estos temas.

\section{Redes que apoyan y promueven el embarazo adolescente}

La familia nuclear es el modelo a seguir de las adolescentes y es la red informal que debe coadyuvar a la prevención del embarazo a temprana edad; sin embargo, la mayoría de las entrevistadas mencionó que no había platicado sobre temas como sexualidad o prevención del embarazo con sus padres antes del embarazo, lo que indica la falta de espacios de confianza. Esto no implica necesariamente una disfunción familiar o núcleos familiares conflictivos; por el contrario, son las discusiones entre los padres o problemas con los hermanos los eventos que pueden generar carencias afectivas que las adolescentes no saben ni pueden resolver, lo que las orilla a tener relaciones sexuales para recibir cierto afecto o a considerar el embarazo como una salida de los problemas familiares.

En las comunidades estudiadas la relación con los padres previa al embarazo fue identificada en la mayoría de los casos como buena (65.5\%), el 17\% indicó que era regular, y el 6.4\% reportó que era mala. Por otra parte, el $79.8 \%$ de las entrevistadas manifestó tener una buena relación con los hermanos, por lo que las malas 
ALEJANDRA PAMELA ESPAÑA-PAREDES,
EL EMBARAZO ADOLESCENTE EN QUINTANA ROO: DEBATES Y APORTES DE INVESTIGACIÓN PARA POLÍTICAS PÚBLICAS DE PREVENCIÓN Y ATENCIÓN relaciones al interior de la familia se desechan como una causa que promueva el embarazo adolescente; después del embarazo manifestaron que las relaciones continuaron siendo buenas, por lo que no se produjeron consecuencias o castigos emocionales por parte de la familia hacia las adolescentes, incluso entre las que se embarazaron por segunda ocasión.

De igual forma, no se modificó su rol dentro del hogar, ya que las labores que realizaban en él permanecieron sin cambio aparente; aumentó la carga laboral, ya que tuvieron que ocuparse no sólo de ellas mismas, sino también de los asuntos del bebé y del esposo o pareja. Algunas respuestas que ilustran esta situación fueron: "ayudar en todo lo que sea de la casa", "encargarme de mi hijo y mi marido", "hacerme responsable de la limpieza y la comida" " "ayudar en casa y también trabajar para mantenerme".

Estas tareas asociadas al plan de vida se vinculan al entender que las responsabilidades de las mujeres aumentaron tras la maternidad; no sólo apoyaban en las labores de la casa, en los casos de las que aún vivían con los padres, sino que también adquirieron la responsabilidad del cuidado del bebé y de la pareja. De esta manera, reproducen el estereotipo de género y a su vez perpetúan el ciclo sin reflexión o cambio de roles.

\section{Educación y proyecto de vida entre las mujeres adolescentes de Quintana Roo}

En México las adolescentes sin escolaridad presentan tasas de fecundidad más altas, entre 180 a 200 por cada mil mujeres, en comparación con las que tienen nivel escolar de secundaria o mayor, con sesenta nacimientos por cada mil mujeres (Villalobos et al., 2015). En el caso de esta investigación, el 52.4\% de las entrevistadas manifestó que estudiaba antes del embarazo, mientras que el $46.8 \%$ afirmó que no. Del grupo que estudiaba, el 27.5\% indicó que continuó estudiando durante su embarazo, mientras que el 61.6\% abandonó la escuela. Asimismo, el 23.6\% mencionó que realizó algún tipo de estudios tras el embarazo, mientras que el 59.4\% no los continuó. Estos datos evidencian que abandonar la escuela es un factor importante para embarazarse a temprana edad, pero también embarazarse en la adolescencia es un factor para abandonar la escuela y para cortar futuras aspiraciones de educación.

Aunado a lo anterior se pudo documentar que existe una relación causa-efecto entre la educación de las madres de las adolescentes y el embarazo de estas últimas; es decir, cuanto menor es la educación de la madre, mayores son las posibilidades de que sus hijas adolescentes se embaracen.

En el capítulo ll.a de la Conferencia de El Cairo se establece que los Estados deben incorporar en los programas de estudios temas relacionados con la salud reproductiva y la igualdad entre los sexos (Naciones Unidas, 1995). Esta información responde al derecho humano a la educación, que a su vez es un requisito sine qua non para asegurar que las personas disfruten de otros derechos humanos como a la salud, a la información y a los derechos sexuales y reproductivos; en otras palabras, la educación sexual integral es parte de los derechos de las y los adolescentes a ser educados en derechos humanos (Amnistía Internacional, 2012). Ante eso, es claro que este derecho sólo se ha ejercido y promovido por el Estado de manera parcial, ya que de los datos obtenidos se desprende que el 71.5\% había recibido pláticas sobre sexualidad y los principales métodos para la prevención del embarazo; únicamente el 26.6\% indicó que no. La escuela fue el lugar donde recibieron la información

Por otro lado, al preguntarles si habían hablado con alguien sobre su sexualidad sólo el 33.4\% respondió afirmativamente, mientras que el $64.9 \%$ indicó que no lo había hecho. Lo anterior revela que la mayoría de las mujeres no consulta sobre sus dudas o temores, lo que les lleva a contar con información parcial.

A pesar de lo anterior, un alto número de mujeres manifestó conocer el condón masculino, el 85\% de las encuestadas - cuatrocientas mujeres- indicó que lo había visto, y el 15\% restante afirmó que nunca lo había visto. Estos porcentajes cambiaron cuando se les preguntó si lo habían usado alguna vez: únicamente el $62 \%$ afirmó haberlo utilizado y el $33 \%$ indicó que no lo había hecho. Los motivos más frecuentes por los cuales no utilizaron el condón fueron: porque no les gustaba 
AlejandRa PAMELA EsPaña-PAREDES, LETICIA JANET PAREDES-GUERRERO, ROCÍO IVONNE QUINTAL-LÓPEZ
EL EMBARAZO ADOLESCENTE EN QUINTANA ROO: DEBATES Y APORTES DE INVESTIGACIÓN PARA POLÍTICAS PÚBLICAS DE PREVENCIÓN Y ATENCIÓN o les producía asco, o por una idea de que, debido a la confianza que le tenían a sus parejas, no quedarían embarazadas.

La mayoría de ellas vivía en unión libre con sus parejas, condición que les dificulta el ejercicio de ciertos derechos a los que sí podrían acceder bajo un matrimonio legal, como los servicios de salud para las familias de los hombres trabajadores. Asimismo, es interesante el hecho de que en un alto porcentaje las parejas de las adolescentes embarazadas, antes de estarlo, no estudiaban, proporción que aumentó tras el embarazo, con un $73 \%$ a $83 \%$ respectivamente, debido a que se incrementóla necesidad de obtener recursos económicos, pero también el rezago educativo en los hombres es un factor para el embarazo adolescente de sus parejas.

De igual importancia es el proyecto de vida de las mujeres adolescentes que viven o vivieron un embarazo y cómo cambió a partir de él, ya que antes del embarazo el $42 \%$ consideraba en su plan de vida estudiar una carrera universitaria, porcentaje que disminuyó tras el embarazo a un 26\%; en lo relativo al trabajo, el 27\% lo visualizaba en su plan de vida y este porcentaje aumentó después a un 34\% debido a la necesidad de obtener recursos económicos para la manutención familiar. Por último, un 22\% de las mujeres después del embarazo contemplaba en su plan de vida únicamente dedicarse a cuidar a su bebé. Sus planes se modificaron significativamente con relación a los que tenían con anterioridad, pues la mayoría de las mujeres consideraba los estudios como una meta a alcanzar, pero después del embarazo éstos quedaron en segundo plano porque el trabajo se volvió una necesidad primordial para la manutención de los hijos. Sin embargo, para algunas de ellas continuar estudiando sigue siendo un factor importante, aunque no logran concretarlo por falta de tiempo, pues la carga de atención que implica el cuidado de los hijos implica para ellas renunciar a sus intereses y poner por delante los de otros.

\section{Conclusiones}

En el presente artículo se exploraron y diferenciaron las experiencias de vida de las mujeres jóvenes del estado de Quintana Roo, situadas en un contexto social y económico específico, con relación a sus embarazos antes de los 20 años, enfatizando aspectos de su educación, vida familiar y plan de vida. Argumentamos que es necesario entender el embarazo en adolescentes no a priori como un problema, sino como una situación de vida y una condición de género.

Hemos visto que desde el enfoque epidemiológico, médico y psicológico el embarazo adolescente ha sido catalogado comúnmente como un problema de salud pública, y ha sido así porque, en efecto, puede derivar en consecuencias graves para la salud de las madres y de los recién nacidos, así como en impactos psicológicos y sociales de largo alcance para ellas y sus familias, que terminan por ponerlas en situación de vulnerabilidad en varios niveles. Sin embargo, es necesario resaltar que para las mujeres entrevistadas el ser madre a temprana edad está naturalizado, pues es lo que han visto y vivido en su entorno cotidiano, por lo que se configura en sus expectativas de vida y de género. Incluso, hay casos en los que las familias y los pares fomentan esta circunstancia debido a que la maternidad es una vía de reconocimiento de la identidad femenina y puede llegar a aliviar la incertidumbre ante la falta de un proyecto de vida claro y optimista. Se observa la necesidad de analizar las situaciones sociales problemáticas desde diferentes ángulos, pero sobre todo de comprender que desde cada óptica se generaran propuestas de atención diferentes. Como se deduce de los resultado del estudio, cuando el embarazo se analiza desde los constructos familiares, personales y de género de las adolescentes, éste no es un elemento problematizador, es más, no se asume como un elemento de vulnerabilidad, sino que se encuentra naturalizado y aceptado en el núcleo familiar.

Respecto a la deserción escolar, en el caso analizado la realidad coincide con los datos estadísticos sobre educación en el país, ya que la mayoría de las entrevistadas abandonó sus estudios después de quedar embarazadas y un número muy reducido logró continuar su proceso educativo después del parto. Incluso, algunas ya habían abandonado sus estudios antes del embarazo. En el presente análisis se observó que la educación de las madres de las adolescentes 
ALEJANDRA PAMELA ESPAÑA-PAREDES,

embarazadas se encontraba en relación proporcional directa con los embarazos de sus hijas, ya que, a menor grado educativo de las primeras, mayor la fecundidad de las segundas. A pesar de que un elemento presente en el proyecto de vida de las entrevistadas era continuar con sus estudios hasta el nivel superior, el contexto de pobreza en el que se desenvolvían limitó el alcance de dicha meta, puesto que la falta de recursos económicos se tornó en un obstáculo crucial para sostener su proceso educativo y derivó en su deserción escolar. Así, una vez alejadas del ámbito educativo y de ese proyecto de vida, la alternativa del matrimonio y de la maternidad temprana pasó a ser una de sus opciones más viables, tal como ocurre en otros contextos anteriormente descritos (Aguilar, 2009; Binstock y Näslund-Hadley, 2010; Stern, 2012).

Como se expuso, el discurso y la visión del Estado parte de que el embarazo adolescente condiciona la escolarización y se convierte en un factor importante de deserción escolar; sin embargo, las estadísticas nacionales y las de este estudio muestran que las adolescentes habían dejado de estudiar antes de quedar embarazadas. Esto indica que son los contextos de falta de oportunidades, pobreza y machismo en el que se encuentran sumergidas, los verdaderos determinantes sociales para la deserción escolar de las jóvenes, y no a la inversa. Evidentemente, la deserción escolar agrava la situación por la que atraviesan las adolescentes, tanto si se produce antes como después del embarazo. Esto se encuentra contemplado con claridad en la Encuesta Nacional para la Prevención del Embarazo en Adolescentes, pero la solución se torna complicada a corto plazo mientras no exista una política pública integral para mejorar las condiciones de vida de la totalidad de los adolescentes en México, y específicamente para ampliar el acceso a todos los grados educativos a nivel económico y cultural, por ser las entidades educativas las unidades básicas donde se imparte educación sexual y los espacios en los que se debe fomentar la construcción de un proyecto de vida para los y las jóvenes, más allá de lo que su condición de género les dicte.
EL EMBARAZO ADOLESCENTE EN QUINTANA ROO: DEBATES Y APORTES DE INVESTIGACIÓN PARA POLÍTICAS PÚBLICAS DE PREVENCIÓN Y ATENCIÓN

Lo mismo se aplica a la transmisión intergeneracional de la pobreza, concepto muy empleado por la academia y el Estado, porque, a pesar de que la pobreza pueda constituir un círculo vicioso, es necesario visualizar que es precisamente ésta la que genera un ambiente vulnerabilizador que fomenta el embarazo en las adolescentes, y no necesariamente a la inversa. En este sentido, las jóvenes entrevistadas ya se encontraban inmersas en situaciones de carencia graves antes de embarazarse y lo único que sucedió tras el embarazo fue la perpetuación de la pobreza económica.

Si bien es cierto que el embarazo en mujeres adolescentes presenta graves riesgos para la salud de la madre y del neonato, este estudio recuerda que no deben perderse de vista las propias concepciones de las jóvenes y sus familias, quienes desconocen los riesgos para la salud existentes en esta condición. Por ello es necesario sensibilizar a los jóvenes sobre los riesgos inmediatos, y a largo plazo para su salud, así como también es necesario informar a los padres de familia para que no sean promotores del embarazo a corta edad, lo que puede poner en riesgo la vida de sus hijas.

En general, con base en los hallazgos se recomienda no considerar a los jóvenes como un grupo homogéneo, estático yfuera de contexto, sino, en cambio, comprender más profundamente la concepción del noviazgo, la sexualidad y el embarazo desde su punto de vista, tomando en cuenta su entorno socioeconómico y sus proyectos de vida, a fin de diseñar programas de prevención del embarazo adolescente más adecuados, así como concretar estrategias para acercar las herramientas de información a los jóvenes en escuelas y centros de salud comunitarios. En segundo lugar, de la mano de lo anterior, es crucial comenzar a transitar desde una política de prevención que ha sido meramente informativa, a una política de promoción de la salud sexual y reproductiva que no aborde el embarazo como "problema" a priori, sino que se centre en construir una noción de salud integral de la mano de los jóvenes. Como varios estudiosos han mostrado, ofrecer información y métodos anticonceptivos no redunda necesariamenteen el aumento de su uso ni en la disminución de embarazos e infecciones de trasmisión sexual (Quintal y Vera, 
AlejandRa PAMELA EsPaña-PAREDES,

2014). Los datos del estudio permiten indicar que se requiere apuntalar la promoción de la salud en diversos terrenos como en sensibilizar y hacer conciencia acerca de la salud, pero también se requiere de trabajar con la construcción de un proyecto de vida y fortalecer los lazos familiares y comunitarios para procurar la salud integral.

En tercer lugar, desde una perspectiva de derechos humanos, es imposible ignorar que cuando en un país se presenta el embarazo o la maternidad a gran escala entre las niñas y adolescentes, como ocurre en México, el Estado está incumpliendo los derechos de niños y niñas y los derechos sexuales y reproductivos de los jóvenes al no garantizarles acceso a información, métodos anticonceptivos y servicios de salud de calidad, así como una oferta educativa accesible. En este sentido, es necesario recordar que una característica fundamental de los derechos humanos es su integralidad. Esto significa que no será posible dar cabal cumplimiento a los derechos sexuales y reproductivos de los jóvenes si no se progresa también en garantizar el derecho a la educación y a una vida libre de pobreza y violencia. Sólo desde un enfoque integral será posible que las cifras de embarazo adolescente comiencen a disminuir de forma significativa, tal y como recomiendan las instancias internacionales. De lo contrario en el país se seguirán destinando millones de pesos a programas cuya efectividad será casi nula.

Por último, las políticas públicas de prevención del embarazo con perspectiva de género no deben perder de vista que el embarazo adolescente es un fenómeno que puede explicarse como una condición de género. Así, a pesar de que se han producido cambios en los contextos socioeconómicos de las mujeres jóvenes, también es necesario avanzar en saldar las brechas de desigualdad de género y en fomentar el empoderamiento femenino para que las jóvenes puedan tener control sobre su proyecto de vida, su desarrollo personal, profesional y laboral y su propio cuerpo, y para que puedan decidir de manera consciente sobre vivir en pareja y la maternidad, tomando en cuenta las implicaciones. El cambio de las leyes no trasforma de manera inmediata las desigualdades sociales acumuladas en las instituciones sociales y la cultura; $\sin$
EL EMBARAZO ADOLESCENTE EN QUINTANA ROO: DEBATES Y APORTES DE INVESTIGACIÓN PARA POLÍTICAS PÚBLICAS DE PREVENCIÓN Y ATENCIÓN

embargo, un abordaje más consciente de las múltiples aristas y variables que inciden en que hoy en día el embarazo adolescente en México aumente cada vez más, en vez de disminuir, es el primer paso para desarrollar políticas públicas pertinentes y efectivas a largo plazo centradas en los grupos más vulnerables.

\section{Notas}

1 De acuerdo con estos datos, el porcentaje de adolescentes en Quintana Roo (23.1\%) que inició vida sexual es ligeramente menor que el nacional (23.0\%) (ENSANUT, 2012).

\section{Referencias}

Aguilar, Paula Lucía (2009). "De silencios y abandonos: La construcción discursiva de la maternidad adolescente en contextos de pobreza". En Revista Latinoamericana de Sexualidad, Salud y Sociedad, 1(3): 130-153.

Amnistía Internacional (2012). “iEs la educación sexual un derecho humano?” Disponible en: https:// redescuelas.es. (consultado el 10 de julio de 2017).

Banco Mundial (2015). "Tasa de fertilidad en adolescentes (nacimientos por cada 1.000 mujeres entre 15 y 19 años de edad)". En Perspectivas de la población mundial. S/l: Banco Mundial. Disponible en: http://datos.bancomundial.org/ (consultado el 27 de junio de 2017).

Binstock, Georgina y Emma Näslund-Hadley (2010). "Iniciación sexual , asistencia escolar y embarazo adolescente en sectores populares de Asunción y Lima : una aproximación”. En Debates En Sociología, 35: 45-67.

Climent, Graciela (2003). "Una expresión de la cuestión social. El interjuego entre la exclusión social, la construcción de la subjetividad y las políticas públicas". En Revista Argentina de Sociología, 1(1): 77-93.

COESPO (Conejo Estatal de Población) (2010). Índices de marginación y rezago social. Chetumal: COESPO.

COESPO (Conejo Estatal de Población) (2011). Anuario estadistico de poblacion. Chetumal: COESPO. 
COESPO (Conejo Estatal de Población) (2013). Perfil de salud reproductiva en Quintana Roo. Chetumal: COESPO. Disponible en: http://coespo.qroo.gob. $\mathrm{mx} /$ de Prensa COESPO Febrero 2013.pdf

CONEVAL (Consejo Nacional de Evaluación de la Política de Desarrollo Social) (2012). Informe de pobreza y evaluación en el estado de Quintana Roo, 2012. México: CONEVAL.

De Jesús, David y Catherine Menkes (2011). "Embarazo y transición a la adultez: un estudio sobre los significados de la sexualidad y la reproducción adolescente en dos contextos de México". En Antonio Marquet (coord.), Hegemonía y desestabilización: diez reflexiones en el campo de la cultura y la sexualidad. México: Ediciones Neon.

ENSANUT (2012). Resultados Encuesta Nacional de Salud y Nutrición de Quintana Roo. México: INSP.

Golovanevsky, Laura (2006). Vulnerabilidad y transmisión intergeneracional de la pobreza: un abordaje cuantitativo para Argentina en el siglo XXI. Buenos Aires: Universidad de Buenos Aires.

INEGI (Instituto Nacional de Geografía e Informática) (2010). Censo de Poblacióny Vivienda 2010. México: INEGI.

INEGI (Instituto Nacional de Geografía e Informática) (2014). Encuesta nacional de la dinámica demográfica 2014. México: INEGI.

INEGI (Instituto Nacional de Geografía e Informática) (2015). "Natalidad y fecundidad". México: INEGI. Disponible en: http://www.beta.inegi.org. $\mathrm{mx} /$ (consultado el 28 de junio de 2017).

Quintal López, Rocío y Ligia Vera Gamboa (2014). "Migración, etnia y género: tres elementos claves en la comprensión de la vulnerabilidad social ante el VIH/SIDA en población maya de Yucatán". En Península, 9(2): 99-130.

Lassonde, Louise (1997). Los desafíos de la demografía: ¿Qué calidad de vida habrá en el siglo XXI? México: CRIM, IIS, PUEG-UNAM, Direction du Développement et de la Coopération de Suiza, Fondo de Cultura Económica.

Naciones Unidas (1995). Informe de la Conferencia Internacional sobre la Población y el Desarrollo. Nueva York: Naciones Unidas.
Naciones Unidas (2015). Objetivos de Desarrollo del Milenio. Informe de 2015. Nueva York: Naciones Unidas. Disponible en: www. http://www. objetivosdedesarrollodelmilenio.org.mx/Doctos/ InfONU2015.pdf

Menkes, Catherine y Leticia Suárez (2003). "Sexualidad y embarazo adolescente en México". En Papeles de Población, 9(35): 233-262. Disponible en: http://www.scielo.org.mx/ (consultado el 5 de marzo de 2018).

Núñez Noriega, Guillermo y Deisy Judith Ayala Valenzuela (2012). "Embarazo adolescente en el noreste de México: entre la tradición y la modernidad". En Culturales, 8(15): 7-46.

OCDE (Organización para la Cooperación y el Desarrollo Económicos) (2017). La luchaporla igualdad de género: una batalla cuesta arriba. Washington: ocde. Disponible en: https://www.oecd.org/mexico/

Okumura, Javier A., Diego A. Maticorena, José E. Tejeday Percy Mayta-Tristán (2014). “Embarazo adolescente como factor de riesgo para complicaciones obstétricas y perinatales en un hospital de Lima, Perú”. En Saúde Mater, 14(4): 383-392.

OMS (Organización Mundial de la Salud) (2014). Embarazo en la adolescencia. S/l: OMS. Disponible en: http://www.who.int/

Rangel, José Luis, L. Valerio, J. Patiño y M. García (2004). "Funcionalidad familiar en la adolescente embarazada". En Revista de La Facultad de Medicina UNAM, 47(1): 24-27.

Reyes, David de Jesús y Esmeralda González Almontes (2014). "Elementos teóricos para el análisis del embarazo adolescente". En Sexualidad, Salud y Sociedad. Revista Latinoamericana, 17: 85-86.

Rosso, Nadia (2016). "El sistema patriarcal: sus fundamentos y funcionamiento". Ponencia presentada en el Diálogo Internacional: Feminicidios en América Latina, organizado por la Fundación Mujer y Futuro en Bucaramanga, Colombia, noviembre de 2016.

Secretaría de Gobernación (2015). Estrategia Nacional para la Prevención del Embarazo en Adolescentes. México: Gobierno de la República. 
Secretaría de Salud (2014). Programa de Acción Específico Salud Sexual y Reproductiva para Adolescentes. México: Secretaría de Salud.

Stern, Claudio (1997). "El embarazo en la adolescencia como problema público: una visión crítica”. En Salud Pública de México, 39(2): 137-143.

Stern, Claudio (2003). "Significado e implicaciones del embarazo adolescente en distintos contextos socioculturales de México". En Estudios Sociológicos, 2l(63): 725-745.

Stern, Claudio (2012). El "problema" del embarazo en la adolescencia. Contribuciones a un debate (lra ed.). México: Colegio de México.

UNESCO (2017). Embarazo precoz y no planificado. Recomendaciones para el sector de la educación. Educación
2030. S/l: UNESCO. Disponible en: http://unesdoc. unesco.org/

Vélez Arango, Alba Lucía (2012). "El embarazo en el adolescente: una visión desde la dimensión emocional y la salud pública". En Revista Cuidarte, 3(1): 394-403.

Villalobos Hernández et al. (2015). "Embarazo adolescente y rezago educativo : análisis de una encuesta nacional en México". En Salud Pública de México, 57(2): 135-143.

Zamudio Raya, César A., Jatziri Silva Contreras, Carlos Gómez Alonso y Paula Chacón Valladares (2017). "Perfil epidemiológico de adolescentes embarazadas adscritas a una unidad de medicina familiar en Morelia, Michoacán, México". En Atención Familiar, 25(2): 54-58. Disponible en:http://www.revistas. unam.mx/. 
ALEJANDRA PAMELA ESPAÑA-PAREDES,

LETICIA JANET PAREDES-GUERRERO, ROCíO IVONNE QUINTAL-LÓPEZ
EL EMBARAZO ADOLESCENTE EN QUINTANA ROO: DEBATES Y APORTES DE INVESTIGACIÓN PARA POLÍTICAS PÚBLICAS DE PREVENCIÓN Y ATENCIÓN

Tabla 1. Matriz multivariada para el estudio de las causas y consecuencias del embarazo adolescente

\begin{tabular}{|c|c|c|c|c|c|c|}
\hline \multicolumn{7}{|c|}{ Variables } \\
\hline & $\begin{array}{c}\text { Caracterización } \\
\text { y entorno }\end{array}$ & Psicológicas & $\begin{array}{l}\text { Biológicas y } \\
\text { de salud }\end{array}$ & Socioculturales & $\begin{array}{l}\text { Económicas } \\
\text { y materiales }\end{array}$ & $\begin{array}{c}\text { Política pública } \\
\text { y servicios }\end{array}$ \\
\hline $\begin{array}{l}\text { Antes del } \\
\text { embarazo } \\
\text { (causa) }\end{array}$ & $\begin{array}{l}\text { Datos generales } \\
\text { Estructura familiar } \\
\text { Pareja } \\
\text { Hijos }\end{array}$ & $\begin{array}{l}\text { Conocimientos } \\
\text { Conducta } \\
\text { Emociones }\end{array}$ & $\begin{array}{l}\text { Salud } \\
\text { Prevención } \\
\text { Adicción } \\
\text { Ejercicio de la } \\
\text { sexualidad }\end{array}$ & $\begin{array}{l}\text { Discriminación } \\
\text { Creencias sobre } \\
\text { la sexualidad y } \\
\text { embarazo } \\
\text { Rol de género } \\
\text { Percepción social } \\
\text { sobre el embarazo } \\
\text { adolescente }\end{array}$ & $\begin{array}{l}\text { Ocupación } \\
\text { Distribución } \\
\text { Manutención }\end{array}$ & $\begin{array}{l}\text { Conocimiento } \\
\text { Uso }\end{array}$ \\
\hline $\begin{array}{l}\text { Después del } \\
\text { embarazo } \\
\text { (consecuencias) }\end{array}$ & $\begin{array}{l}\text { Datos generales } \\
\text { Estructura familiar } \\
\text { Pareja } \\
\text { Hijos }\end{array}$ & $\begin{array}{l}\text { Conocimientos } \\
\text { Conducta } \\
\text { Emociones }\end{array}$ & $\begin{array}{l}\text { Salud } \\
\text { Prevención } \\
\text { Adicción } \\
\text { Ejercicio de la } \\
\text { sexualidad }\end{array}$ & $\begin{array}{l}\text { Discriminación } \\
\text { Creencias sobre } \\
\text { la sexualidad y } \\
\text { embarazo } \\
\text { Rol de género } \\
\text { Percepción social } \\
\text { sobre el embarazo } \\
\text { adolescente }\end{array}$ & $\begin{array}{l}\text { Ocupación } \\
\text { Distribución } \\
\text { Manutención }\end{array}$ & $\begin{array}{l}\text { Conocimiento } \\
\text { Uso }\end{array}$ \\
\hline
\end{tabular}

Fuente: elaboración propia. 
ALEJANDRA PAMELA ESPAÑA-PAREDES,

LETICIA JANET PAREDES-GUERRERO, ROCíO IVONNE QUINTAL-LÓPEZ
EL EMBARAZO ADOLESCENTE EN QUINTANA ROO: DEBATES Y APORTES DE INVESTIGACIÓN PARA POLÍTICAS PÚBLICAS DE PREVENCIÓN Y ATENCIÓN

Tabla 2. Creencias sobre la mujer y el embarazo

\begin{tabular}{lccc}
\hline \multicolumn{1}{c}{ Afirmación } & Cierto $\%$ & Falso \% & No lo sé $\%$ \\
\hline Embarazarte adolescente es malo para la salud & $40.6 \%$ & $47.2 \%$ & $3 \%$ \\
Ser madre a edad temprana no permite seguir estudiando & $28.1 \%$ & $61.7 \%$ & $1.3 \%$ \\
El embarazo adolescente es un problema social & $36.8 \%$ & $52.1 \%$ & $1.5 \%$ \\
La misión de las mujeres es tener hijos & $29 \%$ & $59.8 \%$ & $1.7 \%$ \\
Las mujeres sólo sirven para ser madres & $6.4 \%$ & $83 \%$ & $0.6 \%$ \\
Es responsabilidad de mi pareja cuidarme & $46.2 \%$ & $43.8 \%$ & $0.8 \%$ \\
Antes de embarazarme, creía que los anticonceptivos no servían & $21 \%$ & $67.6 \%$ & $1.3 \%$ \\
Antes de embarazarme, creía que a mí nunca me iba a pasar & $45.1 \%$ & $44 \%$ & $0.4 \%$ \\
Pensé que teniendo una pareja iba a solucionar mis problemas de casa & $19.1 \%$ & $70.4 \%$ & $0.2 \%$ \\
Yo no quería tener relaciones sexuales, me obligaron & $3.8 \%$ & $85.3 \%$ & $0.2 \%$ \\
Sólo quería disfrutar & $33 \%$ & $55.3 \%$ & $0.2 \%$ \\
Nunca pensé en las consecuencias de tener relaciones sexuales & $35.7 \%$ & $53.8 \%$ & $0.4 \%$ \\
\hline
\end{tabular}

Fuente: elaboración propia. 